Usages du français et pratiques d'enseignement en Europe balkanique, centrale et orientale - Grèce,

Serbie, Bulgarie, Moldavie, Hongrie, Allemagne, Russie - XVIIIe - XXe siècles

\title{
L'enseignement du français en Hongrie après le traité de Trianon (1920) : un essor défiant les contingences politiques?
}

Catherine Tamussin

\section{(2) OpenEdition \\ 1 Journals}

\section{Édition électronique}

URL : https://journals.openedition.org/dhfles/4212

DOI : $10.4000 /$ dhfles. 4212

ISSN : 2221-4038

Éditeur

Société Internationale pour l'Histoire du Français Langue Étrangère ou Seconde

Édition imprimée

Date de publication : 1 juin 2015

Pagination : 131-153

ISSN : 0992-7654

\section{Référence électronique}

Catherine Tamussin, «L'enseignement du français en Hongrie après le traité de Trianon (1920) : un essor défiant les contingences politiques? ", Documents pour l'histoire du français langue étrangère ou seconde [En ligne], 54 | 2015, mis en ligne le 01 janvier 2018, consulté le 27 mars 2023. URL : http:// journals.openedition.org/dhfles/4212 ; DOI : https://doi.org/10.4000/dhfles.4212 


\title{
L'enseignement du français en Hongrie après le traité de Trianon (1920) : un essor défiant les contingences politiques?
}

\author{
Catherine Tamussin
}

1 Dans le contexte difficile de l'après Première Guerre mondiale, la Hongrie introduit en 1924 une réforme de l'enseignement secondaire qui permet, pour la première fois dans l'histoire de l'enseignement des langues dans le pays, la mise en place de cours de français, d'anglais et d'italien à côté des cours d'allemand, langue obligatoire depuis la fin du XVIII e siècle. Même si cette orientation visant à équilibrer la suprématie historique de la langue et de la culture germaniques a eu des précédents dans l'histoire magyare, elle peut sembler quelque peu étonnante voire paradoxale à une époque où les relations diplomatiques entre la France et la Hongrie sont très tendues, après la signature, en juin 1920, du traité de paix de Trianon, qui démantèle l'empire d'Autriche-Hongrie. Aussi, notre hypothèse de travail est que l'influence de la culture et de la langue françaises, en dépit de ce contexte difficile et peu propice, a non seulement perduré mais a connu un développement consistant dans la Hongrie de l'entre-deux-guerres, notamment dans l'enseignement secondaire.

2 Après un bref aperçu du contexte historique et social, nous analyserons les effets de la réforme de 1924 sur l'enseignement des langues étrangères et en particulier sur la diffusion du français. Pour cela, nous analyserons les statistiques nationales hongroises (Asztalos 1934), les discours du ministre de l'Instruction publique de l'époque, et nous nous appuierons sur les travaux des historiens hongrois de l'éducation. Enfin, nous verrons les ambiguïtés et les limites de cette réforme tout en évaluant son impact global sur l'essor du français dans la Hongrie des années 1920. 


\section{Un contexte historique et diplomatique peu propice au développement des relations culturelles franco- hongroises}

\subsection{La guerre et le traité de paix de Trianon : points de rupture et catalyseurs de changement}

3 L'Autriche-Hongrie sort vaincue de la Première Guerre mondiale. En 1920, lors de la signature du traité de paix à Trianon, la politique des pays vainqueurs (France, GrandeBretagne, États-Unis, Italie) est de démanteler cet empire en créant, autour d'une Hongrie démembrée, une couronne protectrice de petits états pour faire contrepoids à la puissance germanique.

4 L'éminent historien Carlile Aylmer Macartney, académicien britannique, spécialiste de l'Europe centrale et en particulier de la Hongrie, ouvre ainsi l'étude impartiale qu'il fait dès 1937 du traité de Trianon et de ses possibles conséquences : « Aucun traité de paix n'a été plus drastique dans ses termes que le traité de Trianon ${ }^{1}$ " (Macartney $1937: 1$ ).

En effet, l'ancien Royaume de Hongrie inclus dans l'Empire austro-hongrois, perd plus des deux-tiers de son territoire passant de 325000 à environ $93000 \mathrm{~km}^{2}$. En termes de population, cela représente aussi une perte de plus des deux-tiers de la population : la Hongrie de 1914 comptait environ 20,9 M. d'habitants, elle n'en compte plus que 7,6 M. en 1920. De plus, près de 2,750 M. de Hongrois (Romsics 1999 : 140) se retrouvent hors des nouvelles frontières, créant des foyers de minorités hongroises en Tchécoslovaquie, Yougoslavie, Roumanie, Ukraine et même en Autriche.

6 Avant la guerre, les Hongrois représentaient à peine la moitié de la population du Royaume de Hongrie qui comprenait de nombreuses nationalités : $14 \%$ de Roumains, $10 \%$ d'Allemands, $10 \%$ de Slovaques, $9 \%$ de Croates, $5 \%$ de Serbes, et encore $4 \%$ d'autres peuples (Italiens, Ruthènes, Grecs, etc.). Avec exactement un taux de $48 \%$, les Hongrois n'étaient pas globalement majoritaires, même s'ils l'étaient à l'échelle de certaines régions.

7 Le traité de Trianon crée donc une situation complètement nouvelle, où la Hongrie passe d'un état multinational et plurilingue soumis à la tutelle de l'Autriche, à un état, certes indépendant, mais drastiquement amputé, où la population est quasiment homogène tant au niveau ethnique que linguistique. En 1920, les Hongrois, dans le territoire réduit de la nouvelle Hongrie indépendante, représentent désormais presque $90 \%$ de la population, avec des minorités très peu nombreuses. Seule la minorité allemande, très implantée dans le cœur du pays depuis longtemps, reste importante avec $10 \%$.

8 Ce passé plurilingue très récent a encore un impact sur les compétences linguistiques des élèves, même si celles-ci tendent à diminuer, comme le remarque János Asztalos, auteur des statistiques sur les écoles secondaires :

Les données sur la connaissance des langues étrangères présentaient un aspect plus favorable dans l'ancienne Hongrie, puisqu'une grande partie des élèves avaient autrefois l'occasion d'apprendre la langue des minorités nationales respectives. D'après les données d'autrefois, 30\% des élèves hongrois parlaient aussi d'autres langues, dernièrement cette proportion n'est plus que de 20\%. (Asztalos 1934 : 44) 
Ce passé plurilingue est aussi important pour la connaissance de l'allemand, à la fois langue de minorité nationale et langue étrangère, ce qui lui donne un statut particulier.

Dans le domaine de l'éducation, la réduction du territoire provoque la perte de plus de la moitié des écoles secondaires. Elle entraîne aussi l'exode de 300000 Hongrois des territoires annexés par les pays voisins vers la "petite Hongrie » avec parmi eux un grand nombre d'enseignants, d'avocats et d'intellectuels restés sans travail, qui viennent ainsi accentuer la pénurie d'emplois publics dans un pays désorganisé économiquement suite au démembrement de son territoire. Ces circonstances très difficiles contraignent les autorités à une profonde restructuration.

11 Enfin, le démantèlement de l'Autriche-Hongrie et la chute de la dynastie des Habsbourg a provoqué une déstabilisation politique totale. La Hongrie connait la période la plus troublée de son histoire passant d'une tentative de démocratie républicaine menée par un noble réformiste, le comte Károlyi, à la « révolution prolétarienne » de Béla Kun, à la contre-révolution "blanche", issue du coup de force de l'armée dirigée par l'amiral Horthy, ancien commandant en chef de la flotte austro-hongroise, bénéficiant du soutien des Alliés. Élu régent le $1^{\mathrm{er}}$ mars 1920 , sous la pression des armes de ses partisans qui occupent le Parlement, l'amiral Horthy instaure une monarchie sans roi, situation " provisoire » qui durera plus de vingt ans. Dans ce régime, « au sommet de la classe politique, on trouve l'aristocratie revenue en force, autant sinon plus qu'à l'époque de la monarchie qui avait procédé à une certaine démocratisation de la fonction publique et de l'armée " (Molnár 2004 : 344). Ainsi, la réforme de 1924 sera mise en place par ce gouvernement conservateur qui entre alors dans la phase de stabilisation de son pouvoir et cherche à consolider ses appuis dans la société.

\subsection{Tensions et restrictions au niveau des relations diplomatiques entre la France et la Hongrie}

12 Dans ce contexte du traité de Trianon, les relations diplomatiques franco-hongroises sont très tendues, avec des effets restrictifs sur la politique culturelle de la France visà-vis de la Hongrie: tandis qu'on ouvre des Instituts français en Europe centrale et orientale à Prague (1920), Bucarest (1923), Varsovie (1924), Belgrade (1926), il n'est nullement question d'en créer un en Hongrie (Chevalier 2001: 134). Les consignes données aux diplomates arrivant en poste à Budapest dans les années 1920 se bornent à la stricte surveillance de l'application des clauses du traité de Trianon et à la vigilance quant aux éventuelles velléités irrédentistes hongroises :

$\mathrm{Au}$ moment où vous allez rejoindre votre poste ${ }^{2}[\ldots]$, il me paraît utile de vous indiquer les principes directeurs de la politique à laquelle vous aurez à collaborer [...]. La condition essentielle du maintien de relations normales entre la France et la Hongrie réside dans l'exécution loyale par ce pays des stipulations du Traité de Trianon. Votre première préoccupation devra être en conséquence de surveiller attentivement cette exécution. (MAE, SOFE, Hongrie, 5.417QO/149bis)

Dans ce contexte où la politique française favorable à la Petite Entente laisse peu de marge de manœuvre sur place, il est évident que le poste de Budapest ne figure pas parmi les plus attractifs du réseau diplomatique. D'ailleurs, concernant le personnel, avec un ambassadeur et deux ou trois diplomates, il compte parmi les petits postes (Ablonczy 2000 : 1153). De 1922 à 1927, le nombre important de diplomates se succédant après avoir effectué seulement une courte période d'exercice semble confirmer que 
Budapest n'était pas un poste convoité pour une promotion de carrière (Vrain 2001 : 65). Était-il pour autant aussi ennuyeux ou inintéressant qu'on pourrait le supposer a priori ? À défaut de pouvoir agir au niveau politique vu la fermeté des consignes, n'y avait-il pas moyen pour les diplomates de développer ou d'appuyer des projets au niveau culturel? À cet égard, nous analyserons à travers la correspondance entre Budapest et Paris, comment les diplomates français en poste réagirent au lancement de la réforme de 1924.

\section{La réforme de 1924 : objectifs et moyens}

\subsection{Un objectif clairement avoué : répondre aux besoins d'une certaine classe sociale}

Kuno Klebelsberg, ministre de l'Instruction publique et des Cultes de 1921 à 1931, dans un article du Journal National du 23 août 1924, explique clairement l'objectif de la réforme envisagée dans l'enseignement secondaire :

Pour favoriser la réussite de la classe moyenne hongroise, compte tenu des manques actuels dans les programmes scolaires, je considère qu'un enseignement plus conséquent des langues modernes [...] est nécessaire. Pour cela, je souhaite introduire un nouveau type de lycée: le reálgimnázium. (Gróf Klebelsberg Kuno válogatott beszédei és irásai 1990 : 303)

Quelle est donc cette classe moyenne hongroise? Pourquoi est-elle si importante au point d'être le destinataire principal de cette réforme? En fait, il ne s'agit pas ici de la bourgeoisie, comme dans nos pays occidentaux, mais de la dzsentri. Ce terme emprunté en 1875 à l'anglais gentry désigne les moyens et petits nobles, propriétaires fonciers souvent endettés et appauvris. Dans la hiérarchie sociale, la dzsentri se situe au milieu, entre d'une part la haute aristocratie alliée avec la grande bourgeoisie financière et commerciale, et d'autre part la grande masse des paysans. Par ailleurs, cette classe moyenne n'est pas nettement cloisonnée :

Cet amalgame de la noblesse foncière, du haut fonctionnariat et des roturiers les plus distingués constitue une classe politique et une classe moyenne difficilement définissables, une classe de notables qui s'appellera plus tard «classe moyenne seigneuriale chrétienne ». [...] Il s'agit bien d'une auto-identification spécifiquement hongroise, très différente de la bourgeoisie d'autres pays. (Molnár 2004 : 298-299)

Aurélien Sauvageot ${ }^{3}$, lecteur au Collège Eötvös de 1923 à 1931, décrit ainsi la dzsentri dans son livre Découverte de la Hongrie :

Cette petite noblesse est obsédée par l'ambition d'égaler la grande aristocratie. Elle se saigne pour vivre au-dessus de ses moyens, achevant ainsi de se ruiner. Son unique providence est l'État. Aussi ses fils forment-ils le gros des fonctionnaires [...]. Le pouvoir central sait qu'il peut compter sur leur loyalisme à toute épreuve. (Sauvageot $1937: 48$ )

17 Cette dernière phrase nous révèle l'importance de la dzsentri qui constitue l'assise sociale du pouvoir conservateur en place depuis 1920. À cet égard, il est intéressant de noter que le ministre Kuno Klebelsberg né en 1875 en Transylvanie, est lui-même issu de cette classe sociale. Sa mère venait d'une famille de la petite noblesse hongroise, son père était un aristocrate officier fonctionnaire de l'armée autrichienne. Noble, il a hérité de sa famille le titre de comte mais il n'a ni fortune mobilière, ni terres. 


\section{2 Création d'un nouveau type de lycée privilégiant les langues vivantes étrangères} compromis entre les deux types d'établissements secondaires déjà existants, le gimnázium et la reáliskola.

19 grec. La reáliskola, inspirée du modèle germanique hérité de l'Empire autrichien, dispense, comme son nom l'indique, un enseignement plus pratique, basé sur les matières scientifiques (mathématiques, géométrie, biologie, chimie), sans latin mais avec des langues vivantes étrangères.

Le reálgimnázium sera donc l'établissement qui permettra de combiner latin et langues vivantes étrangères, car :

La classe moyenne historique avait besoin du latin pour renforcer sa légitimité historique face à la bourgeoisie industrielle et commerciale et aux couches sociales inférieures, mais elle avait aussi besoin d'études moins académiques avec des langues modernes. Le régime était dirigé par les aristocrates [...] mais aussi par les industriels capitalistes et tous avaient besoin d'études beaucoup plus pratiques. (Nagy 1999 : 16)

\section{Effets de la réforme sur l'enseignement des langues et en particulier du français}


Nombre d'heures hebdomadaires d'enseignement des langues de la $1^{\text {ère }}$ à la $8^{\mathrm{e}}$ classe selon le type d'écoles secondaires de garçons et de filles ${ }^{4}$ (graphique 1 )

Nombre d'heures hebdomadaires d'enseignement des langues de la lère à la 8 e classe selon le type d'écoles secondaires de garçons et de filles ${ }^{1}$ (graphique 1)

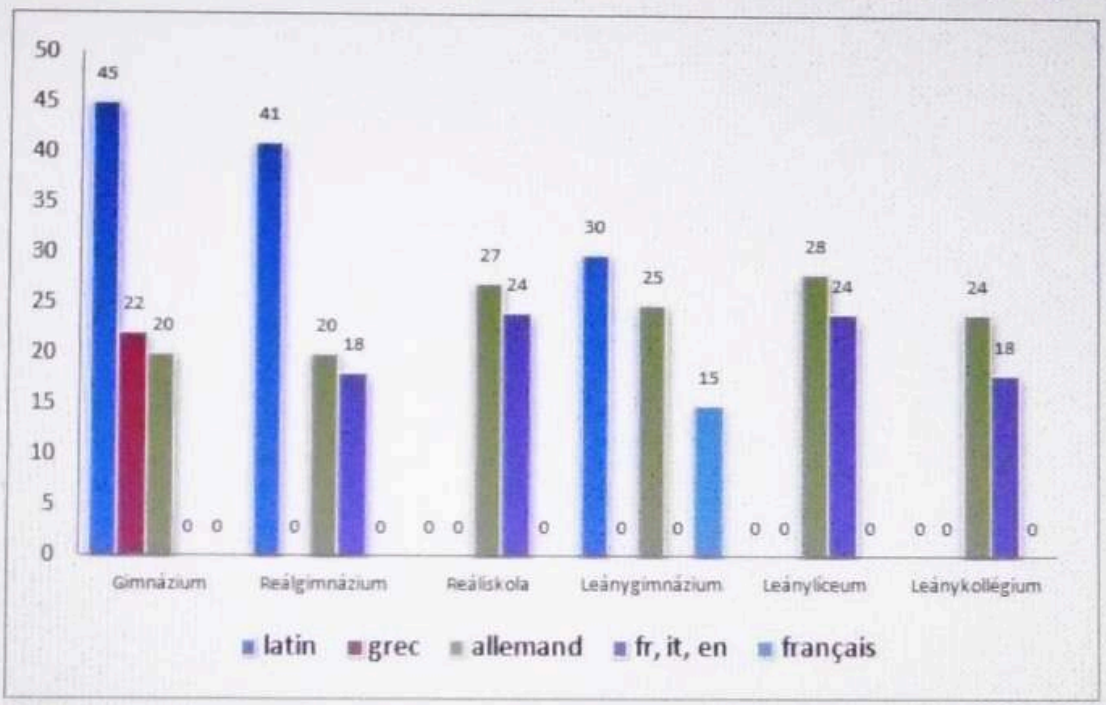

D'après Komis Gyula, Magyarország közoktatásügye a világháborí óta, Magyar Paedagogiai Társaság, Budapest, 1927, p. 103-104 et 162-163

D'APRÈS KORNIS GYULA, MAgYARORSZÁg KÖZOKTATÁSÜgYE A VILÁgHÁBORÚ ÓTA, MAgYAR PAEDAgogIAI TÁRSASÁg, BUDAPEST, 1927, P.103-104 ET 162-163.

\subsection{Permanence de la fonction élitiste du latin}

21 Il ressort de l'analyse du graphique 1 que le latin, sur les huit années de scolarité secondaire, s'avère la langue la plus importante en nombre d'heures dans les trois types de lycées où elle est enseignée à savoir gimnázium (45), reálgimnázium (41) et leánygimnázium (30).

Cette prédominance du latin n'est pas seulement liée à un rôle de formation culturelle humaniste destinée à une élite, comme dans les pays occidentaux. Le latin n'était pas qu'une simple matière enseignée à côté de la langue nationale. Resté très tardivement, jusqu'au milieu du XIX siècle, langue officielle et langue d'enseignement dans le secondaire, il a pu être ainsi un instrument de résistance à la germanisation et revêtait une fonction de langue d'état dans un pays multilingue comme la Hongrie. Utilisé dans le gouvernement, l'administration et la magistrature, le latin est devenu l'expression de la domination de l'élite hongroise, seule capable d'utiliser cette langue officielle et par conséquent d'accéder aux postes dirigeants (Nagy 2000 : Századok, 1325).

Continuer à privilégier le latin équivalait donc à préserver cette fonction de domination d'une élite qui se perpétuait dans la formation typique du jeune aristocrate de la classe moyenne traditionnelle : un baccalauréat classique couplé avec des études de droit afin d'acquérir la langue et les connaissances nécessaires pour accéder à la haute administration et aux hautes fonctions de l'État. D'ailleurs, Kuno Klebelsberg a 
lui-même suivi cette voie en fréquentant le gimnázium de Székesfehérvár pour faire ensuite des études de droit à Budapest, Berlin, Munich et Paris.

\subsection{L'allemand première langue vivante obligatoire : une suprématie non ébranlée}

Le graphique 1 souligne la suprématie incontestable de l'allemand qui, non seulement reçoit le plus grand nombre d'heures (entre 20 et 28) comparé aux autres langues vivantes (entre 15 et 24 heures), mais est enseigné dans les trois types d'établissements secondaires et aussi bien pour les garçons que pour les filles.

La dynamique d'une langue dépend de son statut (juridique et affectif), [...] ainsi que des liens que cette langue entretient avec les autres langues et cultures en présence. Dans cette optique large, l'apprentissage des langues en Hongrie est la résultante de circonstances historiques et culturelles, de choix politiques et de choix individuels. (Szende 2009 : 125)

Cette observation de Thomas Szende nous donne une clé de lecture pertinente pour comprendre le rapport spécifique que les Hongrois entretiennent avec la langue allemande. Les propos du ministre de l'Instruction publique, publiés dans Nemzeti Újság, 23-24 août 1924, illustrent bien cette dynamique :

Parmi les peuples européens, ce sont les Allemands qui géographiquement sont les plus proches de nous, et comme c'est entre peuples voisins que les contacts sont les plus étroits, il faut maintenir la langue allemande comme matière obligatoire à l'avenir aussi. Par le passé, l'allemand fut rendu obligatoire vu son emploi dans l'armée et les institutions communes. Vécu comme une contrainte puisqu'il était imposé, il a inspiré de l'antipathie à beaucoup de Hongrois. Maintenant, c'est dans notre intérêt économique et culturel d'apprendre l'allemand et tout arrière-goût désagréable associé à la contrainte se dissout de lui-même. (Gróf Klebelsberg Kuno válogatott beszédei és irásai 1990 : 304)

Le ministre ressent visiblement le besoin de justifier son choix politique de maintenir la suprématie de l'allemand: comme les circonstances historiques et culturelles remontent à un passé difficile où l'usage de la langue allemande fut imposé par une puissance dominante, il s'agit, selon lui, de transformer une ancienne contrainte en un choix individuel utile et rationnel. En effet, même si on ne peut parler d'une politique consciente de germanisation, certaines mesures relevèrent indéniablement d'une volonté de centralisation, comme la loi Educationis éditée en 1777 par l'impératrice Marie-Thérèse d'Autriche à la fin du XVIII ${ }^{\mathrm{e}}$ siècle, instaurant l'allemand comme matière obligatoire dans les écoles.

Cette place spécifique de la langue allemande en Hongrie est encore renforcée par la présence de minorités allemandes hétérogènes, arrivées dans le pays à des moments différents, avec des origines différentes, des dialectes allemands différents, des statuts sociaux différents. Cette communauté allemande a joué aussi un rôle-clé dans l'industrialisation très concentrée sur Budapest, qui de ce fait, est restée longtemps une ville majoritairement germanophone ${ }^{5}$. À son arrivée en 1923, Aurélien Sauvageot, en tant que français germaniste, ne se sent pas dépaysé dans la capitale, où l'influence germanique est encore bien présente vu que « toute personne tant soit peu instruite y sait l'allemand» et que le quotidien Pester Lloyd, paraît en allemand matin et soir (Sauvageot $1937:$ 15-16). 


\subsection{Le français principal bénéficiaire de la réforme comme seconde langue vivante étrangère}

Dans ce contexte de permanence du latin et de suprématie de l'allemand, qu'en est-il du français? Comme seconde langue vivante, la loi de 1924 prévoit le choix entre anglais, français et italien. Le diagramme suivant permet de voir comment s'est opéré ce choix dans les écoles secondaires de garçons.

Nombre total de lycées de garçons selon leur type. Proportion de ceux où l'on enseigne le français, l'anglais, l'italien en 1932/33 (graphique 2) Nombre total de lycées de garçons selon leur type. Proportion de ceux
où l'on enseigne le français, l'anglais, l'italien en 1932/33 (graphique 2)

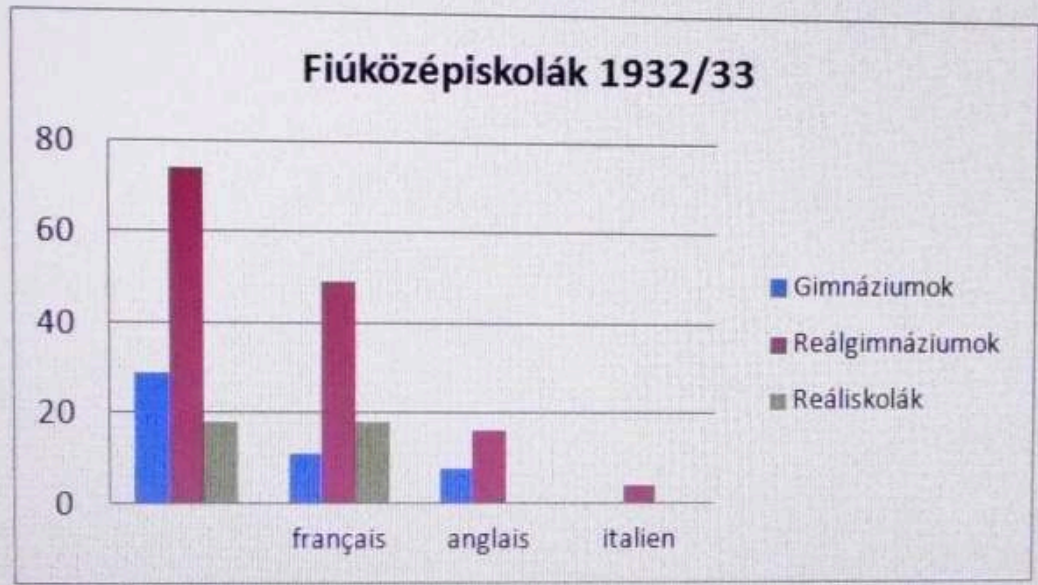

D'après József Asztalos, A magyar középiskolák statisztikája az 1932/33 tanévig, M. Kir. Központi Statisztikai Hivatal, Budapest, 1934, p.42, 44-45 et $22-23$

D’après József Asztalos, A magyar középiskolák statisztikája az 1932/33 tanévig, M. Kir. Központi Statisztikai Hivatal, Budapest, 1934, p. 42, 44-45 et 22-23.

Il apparaît clairement que cette réforme a été très positive pour le français qui est très majoritairement voire exclusivement choisi comme deuxième langue vivante étrangère dans chaque type d'établissements. Ainsi, sur 74 reálgimnázium en 1932/33, environ deux-tiers ont introduit le français soit 49 exactement, 16 l'anglais, 4 l'italien. Dans les reáliskola, le français bénéficie d'une totale exclusivité, il est même institué deuxième langue vivante obligatoire. C'est dans les gimnázium qu'il est moins fortement représenté avec environ 11 lycées sur 29 qui ont introduit le français et 8 l'anglais. Il reste 10 lycées qui ont probablement maintenu l'enseignement du grec ancien et ne proposent donc pas de seconde langue vivante.

Enfin, dans les écoles secondaires de jeunes filles, les statistiques du ministère de l'Instruction publique (Asztalos 1934) révèlent une situation encore plus favorable au français. La réforme, mise en place pour les filles en 1926, a entraîné la création d'un 
grand nombre d'écoles secondaires de filles qui, presque toutes, ont introduit le français comme deuxième langue vivante étrangère, soit 30 écoles sur 44. De plus, dans 22 écoles, le français est seconde langue vivante obligatoire. Dans les écoles secondaires de filles, il y a donc un quasi-monopole du français comme deuxième langue vivante étrangère.

\section{Comment expliquer le succès du français ?}

\section{1 Français langue de culture de niveau international ?}

31 Pourquoi des résultats si positifs? Pourquoi le choix s'est-il porté d'abord sur le français plutôt que sur l'anglais ou l'italien? Dans les préfaces des principaux manuels de français de l'époque, le français y est présenté par les auteurs comme la seule langue de culture de niveau international capable d'être sinon un contrepoids du moins une alternative à la culture allemande dominante en Hongrie. De plus, la richesse de la littérature française exerce un très fort pouvoir d'attraction à une époque où l'accès à la culture se faisait encore essentiellement par l'écrit, et où l'apprentissage de la langue restait basé quasi exclusivement sur la lecture.

\subsection{Un élément décisif : le nombre de professeurs disponibles capables d'enseigner le français}

En vérité, les arguments liés aux représentations mentales sur l'utilité ou l'importance de telle ou telle langue sont inopérants face à la réalité du terrain, si bien que l'élément décisif semble avoir été un aspect bien plus pratique, comme le laissent entendre les propos du ministre :

À côté de l'allemand, on enseignera une deuxième langue moderne dans le nouveau reálgimnázium : l'anglais, le français ou l'italien. Le mieux serait, bien sûr, que dans chaque école il y ait des professeurs pour ces trois langues, de manière à ce que les enfants et les parents puissent choisir l'une d'entre elles. Cette mission incombe au futur, maintenant il faut que j'arrive à avoir assez de bons professeurs pour qu'à côté de l'allemand je puisse faire assurer avec succès l'enseignement d'une deuxième langue moderne. (Gróf Klebelsberg Kuno válogatott beszédei és irásai 1990 : 304)

Le facteur déterminant semble donc avoir été le nombre de professeurs capables d'enseigner telle ou telle langue au moment du lancement de la réforme. Or, vu les résultats très positifs obtenus, on peut logiquement en induire que les professeurs capables d'enseigner le français étaient beaucoup plus nombreux que pour les autres langues, ce qui est somme toute significatif du rayonnement de la culture et de la langue française dans les milieux intellectuels hongrois de l'époque. À cet égard, le Collège Eötvös a sans nul doute joué un rôle fondamental.

\subsection{La tradition francophile du Collège Eötvös et son rôle-clé dans la formation des professeurs de français du secondaire}

La fondation en 1895 du Collège Eötvös sur le modèle de l'École normale supérieure constitue un précédent historique d'une politique visant à s'appuyer sur une tradition française pour contrebalancer l'influence germanique prévalant dans les milieux 
universitaires. Fonctionnant comme un internat rattaché à l'Université de Budapest, offrant des cours complémentaires de très haut niveau, le Collège accueille des étudiants bacheliers triés sur le volet. Dans l'entre-deux-guerres, il a joué un rôle essentiel dans la formation d'une élite intellectuelle francophone et francophile, en particulier parmi les professeurs du secondaire et du supérieur (Karady 1985 : 251-252), ces derniers formant à leur tour d'autres professeurs de français. Il est d'ailleurs intéressant de noter que la majorité des étudiants sortant du Collège faisait carrière comme professeur dans le secondaire, contrairement aux normaliens français :

De 1895 à 1945, 730 étudiants ont obtenu leur diplôme. Parmi eux, 115 sont devenus professeurs dans le supérieur, 60 chercheurs, 25 travaillent dans quelque ministère, 18 à l'étranger, 58 sont devenus directeurs d'établissements secondaires. La majorité d'entre eux soit 400, en accord avec les objectifs du fondateur du Collège, Loránd Eötvös, sont devenus professeurs dans le secondaire tandis que 20 se sont engagés dans des carrières littéraires ou artistiques. (Garai 2014 : 9)

De plus, nombre de professeurs de français ayant joué un rôle très important durant la période étaient issus du Collège Eötvös. Citons à titre d'exemple Géza Bárczi, professeur de français de 1920 à 1941 au lycée pilote de Budapest, donc formateur d'enseignants de français mais aussi auteur de manuels de français réédités jusqu'en 1945 (Szathmári 1985) ; Albert Gyergyai (Horváth 2015) et Sándor Eckhardt (Revel 2001), professeurs de français à l'université de Budapest ; enfin, János Hankiss, professeur à l'université de Debrecen (Gorilovics 2007).

L'orientation francophile du Collège n'a pas décliné avec la guerre. Elle a même connu son plus grand essor dans les années 1920 du fait que les normaliens nommés comme lecteurs ${ }^{6}$ de français au Collège avaient aussi un rôle important auprès de la Légation de France, qui les employait dans des tâches relevant d'un attaché culturel. Enfin, cet attachement à la culture française relève parfois du facteur humain et donc de l'affectif aussi :

Il était touchant de voir combien le premier directeur du Collège Eötvös ${ }^{7}[. .$.$] était$ attaché à la culture française. Les livres français avaient l'honneur du maroquin, les livres allemands devaient se contenter de la demi-toile. [...] Apprendre le français était obligatoire pour les élèves de toute spécialité, car [...] selon l'axiome du baron Eötvös même la plus forte dose de culture française ne saurait nuire à la civilisation nationale, alors que l'on sait le danger mortel de l'infiltration de l'esprit germanique. (Eckhardt 1947)

\subsection{Un rôle actif des diplomates et lecteurs français en poste à Budapest malgré les consignes restrictives de Paris}

Dans la marge de manœuvre étroite qui leur est accordée, les diplomates français en poste au moment du lancement de la réforme déploient tous leurs efforts pour soutenir les initiatives hongroises. Même si le travail du diplomate consiste bien à plaider la cause du pays où il est en poste pour obtenir des crédits, la correspondance entre Budapest et Paris dénote une attitude qui va au-delà de ce formalisme et laisse transparaître un investissement personnel d'énergie.

Ainsi, en février 1924, c'est le lecteur Jean Mistler d'Auriol (dont on apprend qu'il entretient « des relations personnelles très étroites ${ }^{8}$ " avec le comte Klebelsberg) qui assume le rôle de relais en transmettant sous forme de note, à la Légation de France, la requête du ministre hongrois de l'Instruction. Ce dernier demande le soutien de la France par l'envoi de cinq ou six professeurs natifs auxquels il prévoit d'accorder un 
traitement hongrois en espérant que la France complètera. La réponse tarde sûrement à venir puisque le diplomate Carbonnel se donne la peine d'agir à deux niveaux au Quai d'Orsay : par un courrier officiel à la Section des écoles et par une lettre semi-officielle écrite à la main sur papier en tête de la Légation de France et commençant par « Mon cher ami ». De ce fait, le destinataire n'est pas spécifié, mais on peut supposer qu'il s'agit du directeur de la Politique commerciale. Dans cette lettre, Carbonnel prend soin de préciser :

Je sais que la question relève des écoles9 à qui j'écris par ce courrier mais je vous la recommande en même temps parce que c'est vraiment bien important au point de vue politique et cela d'autant plus que sur les autres terrains nous n'avons pas grand-chose à offrir. (MAE, SOFE, Hongrie, 5.417Q0/149bis)

Par ailleurs, dans la lettre officielle aux écoles, il met en post-scriptum :

P.S. Je me permets d'insister tout spécialement pour que les sacrifices nécessaires soient faits pour mettre à profit les dispositions actuelles du Ministre hongrois grâce auxquelles il dépend actuellement de nous de développer notre influence par l'enseignement du français dans les écoles officielles. (MAE, SOFE, Hongrie, $5.417 \mathrm{QO} / 149 \mathrm{bis})$

Au final, il obtiendra la venue de trois professeurs natifs affectés comme lecteurs à l'université et dans un lycée.

\section{Limites et ambiguïtés de cette réforme}

\subsection{L'acquisition des langues étrangères n'est pas l'apanage de l'école}

41 Connaissance des langues étrangères selon le type d'école secondaire en 1932/33 (graphique 3) 


\section{Connaissance des langues étrangères selon le type d'école secondaire en 1932/33 (graphique 3)}

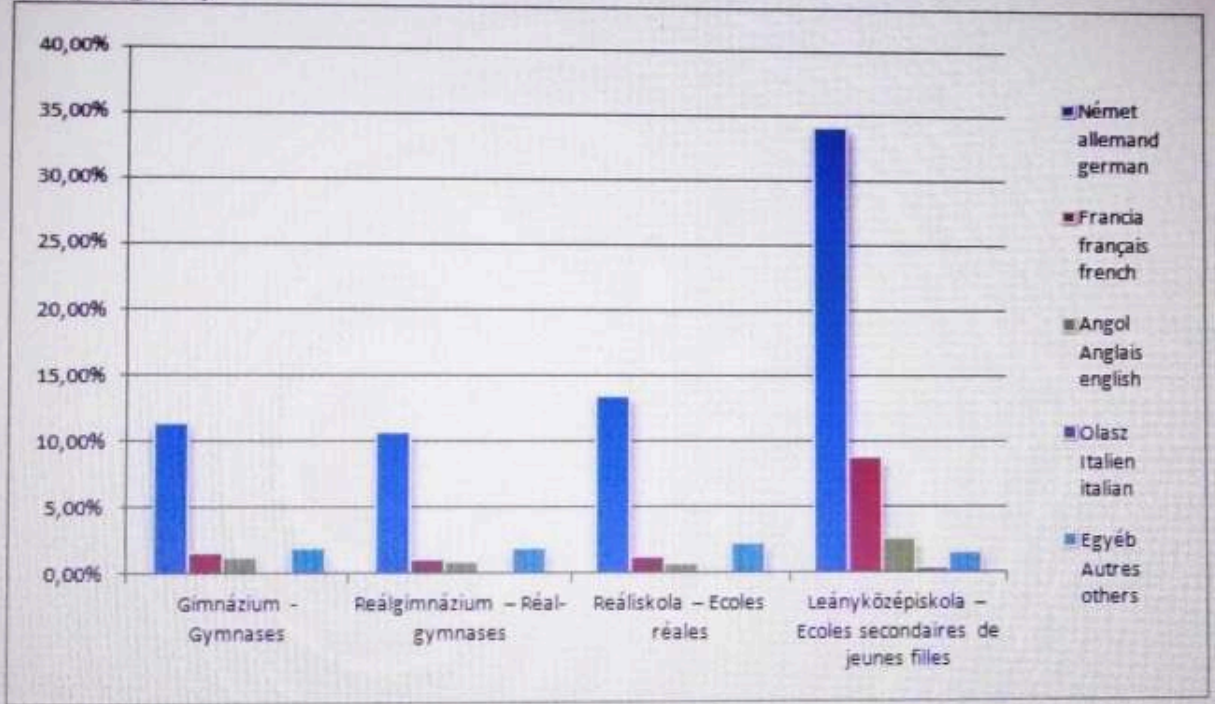

D'après József Astalos, A magyar középiskolák statisztikája az 1932/33 tanévig, M. Kir. Központi Statisztikai Hivatal, Budapest, 1934.

D’après József Astalos, A magyar középiskolák statisztikája az 1932/33 tanévig, M. Kir. Központi Statisztikai Hivatal, Budapest, 1934.

En comparant ce graphique 3 au graphique 1, on remarque qu'il n'y a pas correspondance entre la connaissance d'une langue étrangère et le nombre d'heures d'enseignement de cette langue au lycée.

Par exemple, le français et l'anglais ne figurent même pas comme langues vivantes enseignées au gimnázium pour garçons, mais c'est là qu'est leur meilleur score avec presque $2 \%$ pour le français et environ $1,5 \%$ pour l'anglais.

Un autre cas flagrant est celui des jeunes filles : elles ne reçoivent pas plus d'heures de français que les garçons avec 24 heures dans les leánylíceum, voire même moins avec juste 15 heures dans les leánygimnázium, et pourtant ce sont elles qui battent tous les records de connaissance du français et même de l'anglais avec respectivement presque $10 \%$ et $2,5 \%$ d'entre elles qui parlent ces langues.

La connaissance du français n'a donc qu'un rapport indirect avec le nombre d'heures reçues au lycée et dépend plutôt du niveau social des élèves, comme le souligne l'historien Péter Tibor Nagy : « Plus la composition sociale est 'élégante', plus le nombre d'élèves sachant même les langues non enseignées au lycée est élevé » (Nagy 2000 : 758).

Ce constat renvoie à la pratique traditionnelle de l'élite aristocratique, imitée en cela par la haute bourgeoisie, d'éduquer leurs enfants souvent dès leur plus jeune âge à l'apprentissage du français et d'autres langues en prenant une gouvernante ou un précepteur :

L'aristocrate est de tous les Hongrois celui qui est le moins étroitement attaché au terroir national. Tout enfant, il apprend à manier les langues étrangères. Il arrive même que le hongrois ne soit pas sa langue maternelle, mais seulement un idiome 
dont il fait l'apprentissage après coup, au cours de son adolescence. (Sauvageot $1937: 42)$ privés: «À Budapest, toute une population de Français et de Françaises vit de l'enseignement privé du français. Quelques-uns (et quelques-unes) s'y sont fait une situation que pas mal de braves bourgeois de chez nous leur envieraient » (Sauvageot $1937: 15)$.

\subsection{La réforme touche seulement une élite}

La fonction élitiste des études secondaires est valable partout en Europe à la même époque. De ce point de vue, la Hongrie ne fait pas exception, elle s'inscrit même dans la moyenne européenne avec moins de 3\% de la classe d'âge 10-18 ans faisant des études secondaires en 1914, 5\% environ dans les années 1920 et 10\% à la fin des années 1930 . En revanche, ce qui caractérise la Hongrie, est que cet élitisme émane d'une volonté politique clairement affichée et s'applique essentiellement à une classe sociale en déclin cherchant à maintenir sa position dominante. Ainsi, les statistiques nationales montrent que le nombre de lycéens issus de la gentry passe de 43 à $58 \%$ du total de 1920 à 1938 tandis que les élèves issus de la bourgeoisie (commerce, banque, industrie...) baissent de 28 à $19 \%$, les fils de paysans ne représentant qu'à peine $1 \%$ des élèves (Dékán 1961). Ce favoritisme est également manifeste dans l'attribution des bourses d'études secondaires mises en place à partir de 1927 par le ministère de l'Instruction publique : les principaux bénéficiaires sont fils de hauts fonctionnaires de l'État et de l'armée ou de petits et moyens fonctionnaires. Un seul fils d'ouvrier obtient une bourse.

\subsection{L'intérêt pour les langues vivantes étrangères se développe dans le cadre d'une politique culturelle néonationaliste}

Dans sa réforme éducative, le ministre Kuno Klebelsberg accordait un rôle central à l'enseignement des langues vivantes étrangères. Toutefois, il faut aussi replacer ce rôle dans le cadre d'une idéologie qu'il a lui-même forgée et qualifiée de néonationaliste, et qui s'appuie sur le concept de supériorité culturelle hongroise. En fait, ce concept n'est pas nouveau. L'expression hongroise de kultúrfölény ou supériorité culturelle remonte au milieu du XIX ${ }^{e}$ siècle et se réfère à la situation des Hongrois vivant dans les zones où ils étaient effectivement mêlés à d'autres peuples, les Roumains, les Serbes, les Slovaques et les Ruthènes tous plus pauvres et moins alphabétisés qu'eux, à l'exception cependant des Allemands saxons. La nouveauté, d'où le vocable néonationalisme, est que Kuno Klebelsberg applique ce concept à la nouvelle situation issue du traité de Trianon. La culture devient alors un moyen d'action nationale pour regrouper la magyarité dispersée et un instrument d'influence auprès des puissances occidentales en dotant la Hongrie d'une élite formée aux langues étrangères et donc capable de négocier à un haut niveau. C'est une politique très ambivalente qui amène le ministre à déclarer: «Je voudrais porter à la connaissance générale, que dans la Hongrie démilitarisée suite à la paix de Trianon, le budget du ministère de l'Instruction publique et des Cultes est aussi de fait budget de défense » Gróf Klebelsberg Kuno válogatott beszédei és irásai 1990: 516). De plus, pour asseoir sa légitimité, le régent Horthy saura exploiter le sentiment de frustration nationale issu du traité de Trianon: il accentuera les mesures de nature antisémite ${ }^{10}$ et orientera de plus en plus sa 
politique étrangère vers l'Allemagne, espérant son soutien dans la révision des frontières.

\section{Conclusion}

50 En conclusion, nous reprendrons l'expression de l'historien de l'éducation László Tökéczki qui parle de « réforme conservatrice avec les moyens les plus modernes » (Tökéczki 1990 : 48). En effet, cette réforme éducative use d'instruments très modernisateurs comme l'introduction des langues vivantes étrangères, mais elle s'inscrit dans les limites de la société de l'époque fonctionnant sur le principe féodal conservateur qui consistait à privilégier les intérêts de la classe sociale dominante issue de la petite et moyenne noblesse.

51 L'action même du ministre de l'Instruction et des Cultes, Kuno Klebelsberg, est tout empreinte de paradoxes. Surprenant mélange de modernité, de féodalisme et de néonationalisme, elle reflète d'une part, une grande culture, une vision moderne de l'enseignement des langues, avec des éléments parfois très novateurs comme l'appel à des professeurs natifs selon un dispositif repris sous certains aspects après les changements politiques de 1989. D'autre part, elle reflète un mode de pensée conservateur inscrit dans une logique de reproduction des élites et de solidarité de classe qui fait de Kuno Klebelsberg l'éminent représentant d'une classe sociale en décadence qui cherche à restaurer ses anciennes prérogatives dans une société déstabilisée par la guerre et démembrée par le traité de Trianon.

Cependant, même limitée au cercle restreint d'une élite, cette réforme constitue un tournant décisif car elle introduit les langues vivantes étrangères dans le système éducatif public alors que jusqu'ici précepteurs, gouvernantes ou professeurs privés détenaient le quasi-monopole de cet enseignement. Certes, la motivation reste toujours la même: "Maintenant, où la classe moyenne ne peut plus employer de Fräulein, de Mademoiselle et de Miss pour ses enfants, c'est à l'école de prendre en charge la tâche de l'enseignement des langues modernes » (Gróf Klebelsberg Kuno válogatott beszédei és irásai 1990 : 323).

53 Toutefois, le français est le principal bénéficiaire de cette réforme qui confirme la prédominance de l'allemand mais qui permet une très large diffusion du français comme deuxième langue vivante étrangère la plus enseignée dans les écoles secondaires. Avant la réforme, en 1922-23, sur 38000 élèves, seulement 1517 étudiaient une deuxième langue vivante étrangère. En 1932-33, sur 65328 lycéens et lycéennes, 43552 apprenaient le français soit plus des deux-tiers (Nagy 2000 : 1325).

54 Enfin, cette réforme révèle le fort degré d'influence de la langue et de la culture française au sein d'une élite ainsi que le rôle actif des diplomates français en poste à Budapest malgré des consignes très strictes venant de Paris. 


\section{BIBLIOGRAPHIE}

\section{Sources primaires}

Archives du ministère des Affaires étrangères (MAE), Service des œuvres françaises à l'étranger (SOFE), Hongrie, 5.417QO/149bis.

Archives du ministère des Affaires étrangères (MAE), EUROPE - Hongrie - Correspondance Politique et Commerciale (CPCOM), 3.94 CPCOM/65.

ASZTALOS, József (1934). A magyar középiskolák statisztikája az 1932/33 tanévig. [Statistiques des écoles secondaires hongroises jusqu'en 1932/33]. Budapest : M. Kir. Központi Statisztikai Hivatal.

Gróf Klebelsberg Kuno válogatott beszédei és irásai. [Écrits et discours choisis du comte Kuno Klebelsberg]. (1990). Budapest : Európa Könyvkiadó.

\section{Sources secondaires}

ABLONCZY, Balázs (2000). « Francia diplomaták Magyarországon 1920-1934 » [Diplomates français en Hongrie 1920-1934]. Századok, 134/5, 1149-1170.

CHEVALIER, Jean-Claude (2001). « Diffusion du français en Europe de l'Est : 1920-1939 ». In MarieChristine Kok Escalle et Francine Melka (dir.). Changements politiques et statuts des langues, Histoire et épistémologie 1780-1945 (Actes du colloque de la SIHFLES, Utrecht 1999). Amsterdam : Rodopi, 129-143.

DÉKÁN, Károly (1961). « A középiskolai tanulók szociális megoszlása és ösztöndíjazása a Horthyrendszerben » [Répartition sociale des élèves du secondaire sous Horthy]. Pedagogiai Szemle, $1961 / 2,137-146$.

ECKHARDT, Sándor (1947). Le Collège Eötvös. Budapest : Édition de l'Amicale des anciens élèves du Collège Eötvös.

GARAI, Imre (2014). A tanári elitképzés mühelye. A Báró József Collegium története 1895-1950 [Le collège Eötvös de 1895 à 1950 : un atelier de professeurs d'élite]. Budapest : Eötvös Collegium.

GORILOVICS, Tivadar (2007). « Tanítványból munkatárs, munkatársból barát » [sur János Hankiss]. In Hangodi Ágnes (dir.). A jövő a múlt és a jelen egységére épül. Debrecen : Egyetemi és Nemzeti Könyvtár, 132-141.

HORVÁTH, László (2015). Alulírott, dr. Szegő Albert, írói nevemen Gyergyai Albert [sur Albert Gyergyai]. Budapest : Eötvös József Collegium.

KARADY, Victor (1985). « Le collège Eötvös et l'École normale supérieure vers 1900 ». In Le Goff Jacques et Köpeczi Béla (dir.). Intellectuels français-Intellectuels hongrois. Paris : CNRS, 235-253.

MACARTNEY, Carlile Aylmer (1937). Hungary and her successors: The treaty of Trianon and its consequences 1919-1937. Oxford : Oxford University Press.

MOLNÁR, Miklós (2004) [1980]. Histoire de la Hongrie. Paris : Perrin.

NAGY, Péter Tibor (1999). « The outlines of Hungarian education 1500-1945 ». Educatio, 1999/1, 3-19. 
NAGY, Péter Tibor (2000). « Nyelvpolitika és elitképzés » [Politique linguistique et formation des élites]. Educatio, 2000/2, 743- 760.

NAGY, Péter Tibor (2000). «A 20-as évek középiskola-politikájának kialakulása » [Politique scolaire des années 1920]. Századok, 2000/6, 1313-1334.

REVEL, Jacques (2001). « Alexandre Eckhardt ». In Sahin-Tóth Péter (dir.). Rencontres intellectuelles franco-hongroises. Budapest : Institut français/Collegium Budapest, 251-263.

ROMSICS, Ignác (1999). Magyarország története a XX. században [Histoire de la Hongrie au XX siècle]. Budapest : Osiris Kiadó.

SAUVAGEOT, Aurélien (1937). Découverte de la Hongrie. Paris : Librairie Félix Alcan.

SAUVAGEOT, Aurélien (1988). Souvenirs de ma vie hongroise. Budapest : Corvina.

SZATHMÁRI, István (1985). Bárczi Géza, Budapest : Akadémiai Kiadó.

SZENDE, Thomas (2009). « Politique et linguistique en Hongrie : Quelle place pour les langues européennes? Quelle place pour le français? ». In Thomas Szende (dir.). Politiques linguistiques, apprentissage des langues et francophonie en Europe centrale et orientale. Paris : Éditions des archives contemporaines, 125-132.

TÖKÉCZKI, László (1990). « Konzervatív reform a legkorszerűbb eszközökkel » [Réforme conservatrice avec les moyens les plus modernes]. Valoság, 1990/2, 48-63.

VRAIN, Cécile (2001). « Les diplomates français en poste à Budapest entre 1924 et 1931 ». In Marie Payet \& Ferenc Tóth (dir.). Mille ans de contacts. Relations franco-hongroises de l'an mil à nos jours. Szombathely : Département de français de l'École supérieure Dániel Berzsenyi Szombathely.

\section{NOTES}

1. Les passages des textes anglais et hongrois cités dans cette étude sont traduits par l'auteur (CT).

2. Lettre du ministre des Affaires étrangères à M. De Carbonnel, Ministre plénipotentiaire de la République Française à Budapest, 22 mars 1924. Ces instructions resteront inchangées pendant toute la période de l'entre-deux-guerres.

3. Aurélien Sauvageot (1897-1988) éminent linguiste français est le fondateur des études finnoougriennes en France. En 1923, il est élève à l'École normale supérieure lorsque son maître Antoine Meillet l'envoie à Budapest pour enseigner le français au Collège Eötvös. À son retour en France, il publie en 1937 Découverte de la Hongrie, "œuvre d'un linguiste qui a séjourné en Hongrie de 1923 à 1931 et a commencé à s'initier aussi complètement que possible à la langue du pays. Vivant dans le cadre de l'Université hongroise, au milieu de collègues, d'élèves et d'amis hongrois, il a essayé de s'adapter aussi totalement qu'il a pu à la civilisation hongroise » (Sauvageot $1937: 8$ ). Ce livre est pour nous un témoignage précieux reflétant le regard d'un jeune intellectuel français de gauche qui, du fait qu'il parlait couramment hongrois, a pu s'intégrer dans la vie du pays.

4. En 1926, sont créés pour les filles trois types de lycées (leánygimnázium, leánylíceum, leánykollégium) calqués plus ou moins sur le modèle instauré pour les garçons en 1924.

5. Au milieu du XIX siècle, les deux-tiers, en 1880 un tiers des habitants de la capitale se déclarent de langue maternelle allemande. En 1910, leur proportion n'est plus que de $9 \%$, signe d'une forte assimilation.

6. Jean Mistler d'Auriol (de 1921 à 1923), Aurélien Sauvageot (de 1923 à 1931), François Gachot $(1925 / 26)$. 
7. Géza Bartoniek, directeur de 1895 à 1927.

8. (MAE, SOFE, Hongrie, 5.417QO/149bis), lettre du chargé d'affaires de France en Hongrie à son exc. M. Raymond Poincaré, ministre des Affaires étrangères, 18 février 1924.

9. Il s'agit ici de la Section des écoles du Service des œuvres françaises à l'étranger (SOFE) au ministère des Affaires étrangères.

10. Dès 1920, une loi de numerus clausus limite l'accès de la communauté juive à l'université. Instaurée dans le contexte des exodes de populations hongroises venant des territoires perdus par le traité de Trianon, elle reflète la crainte du manque de débouchés professionnels pour les couches aristocratiques hongroises dirigeantes.

\section{RÉSUMÉS}

En 1924, la réforme de l'enseignement secondaire permet, pour la première fois en Hongrie, la mise en place de cours de français, d'anglais et d'italien à côté des cours d'allemand, langue obligatoire depuis la fin du XVIIIe siècle. Cette politique éducative visant à contrebalancer la suprématie historique de la langue germanique dans le pays, conduira à un essor spectaculaire du français dans les écoles secondaires de garçons et surtout de jeunes filles : plus des deux-tiers des élèves ont choisi le français comme deuxième langue vivante après l'allemand, qui reste prédominant. Certes, cette réforme n'a touché qu'une élite issue de la petite et moyenne noblesse hongroise alliée à la moyenne bourgeoisie. Elle est cependant révélatrice de l'influence de la langue et de la culture françaises au sein de cette élite malgré de fortes tensions diplomatiques entre la France et la Hongrie, suite au traité de paix signé à Trianon réduisant de deux-tiers le territoire de la Hongrie vaincue. Elle montre aussi le rôle actif des diplomates français en poste à Budapest malgré les consignes très strictes venant de Paris.

In 1924, Hungary launches a reform in secondary education which leads to introduce modern languages such as English, French and Italian. For the first time in its history, Hungary attempts to balance the supremacy of German language which was compulsory at school since the 18th century. Actually, Hungarian national statistics show that more than two-thirds of the pupils chose to learn French as a second foreign language after German, which still remained predominant. However, this reform affected only the élite stemming from Hungarian dominant upper-middle aristocracy and bourgeoisie. Still it shows the commitment to French language and culture even if there were deep diplomatic tensions between France and Hungary after the signature in 1920 of the Peace Treaty of Trianon, which reduced drastically the territory of defeated Hungary. Moreover, French diplomats in Budapest supported actively the reform in spite of very strict instructions from Foreign Office in Paris.

\section{INDEX}

Mots-clés : Hongrie, entre-deux-guerres, traité de Trianon, enseignement du français, enseignement des langues, enseignement secondaire, diplomatie culturelle, politique éducative.

Keywords : Hungary, between the wars, treaty of Trianon, teaching French, teaching languages, secondary education, cultural diplomacy, educational policy. 
AUTEUR

CATHERINE TAMUSSIN

INALCO- PLIDAM, Paris 